\title{
Direct single molecule measurement of TCR triggering by agonist PMHC in living primary T cells
}

\author{
Geoff P O'Donoghue ${ }^{1,2+}$, Rafal M Pielak ${ }^{1,2+}$, Alexander A Smoligovets ${ }^{1,2,3}$, \\ Jenny J Lin ${ }^{1,2}$, Jay T Groves ${ }^{1,2 *}$ \\ 'Department of Chemistry, Howard Hughes Medical Institute, University of California, \\ Berkeley, Berkeley, United States; ${ }^{2}$ Physical Biosciences Division, Lawrence Berkeley \\ National Laboratory, University of California, Berkeley, Berkeley, United States; \\ ${ }^{3}$ Department of Molecular and Cell Biology, University of California, Berkeley, \\ Berkeley, United States
}

*For correspondence: JTGroves@lbl.gov

${ }^{\dagger}$ These authors contributed equally to this work

Competing interests: The authors declare that no competing interests exist.

Funding: See page 14

Received: 26 March 2013

Accepted: 17 May 2013

Published: 03 July 2013

Reviewing editor: Axel T Brunger, Howard Hughes Medical Institute, Stanford University, United States

(c) Copyright O'Donoghue et al. This article is distributed under the terms of the Creative Commons Attribution License, which permits unrestricted use and redistribution provided that the original author and source are credited.

\begin{abstract}
T cells discriminate between self and foreign antigenic peptides, displayed on antigen presenting cell surfaces, via the TCR. While the molecular interactions between TCR and its ligands are well characterized in vitro, quantitative measurements of these interactions in living cells are required to accurately resolve the physical mechanisms of TCR signaling. We report direct single molecule measurements of TCR triggering by agonist PMHC in hybrid junctions between live primary T cells and supported lipid membranes. Every pMHC:TCR complex over the entire cell is tracked while simultaneously monitoring the local membrane recruitment of ZAP70, as a readout of TCR triggering. Mean dwell times for PMHC:TCR molecular binding of 5 and $54 \mathrm{~s}$ were measured for two different PMHC:TCR systems. Single molecule measurements of the PMHC:TCR:ZAP70 complex indicate that TCR triggering is stoichiometric with agonist PMHC in a 1:1 ratio. Thus any signal amplification must occur downstream of TCR triggering.
\end{abstract}

DOI: 10.7554/eLife.00778.001

\section{Introduction}

An essential aspect of adaptive immunity is the ability of T cells to discriminate between structurally similar agonist and non-stimulatory self peptides bound to major histocompatibility complex (pMHC) molecules presented on the surface of antigen-presenting cells (APCs) (Weiss and Littman, 1994). Fewer than 10 agonist pMHC molecules can trigger T cells (Irvine et al., 2002; Manz et al., 2011), and the signaling response occurs on a timescale of seconds (Huse et al., 2007). It has been recognized for decades that subtle, peptide-specific differences in binding parameters, especially kinetic rates for the $\mathrm{pMHC}$ and $\mathrm{T}$ cell receptor (TCR) reaction, seem to be the basis of antigen recognition (Matsui et al., 1994; Tian et al., 2007). Experimental correlations between molecular pMHC:TCR binding kinetics, typically determined from solution assays of soluble extracellular domains and bulk measurements of $T$ cell activity, have provided the foundation for much of this understanding.

From a physical point of view, the extreme sensitivity, selectivity, and apparent immunity to stochastic noise exhibited by TCR antigen recognition pose challenges to classical notions of ligand-receptor signaling (van der Merwe and Dushek, 2011; Dustin and Groves, 2012). For example, the maximum affinity a TCR can have for self pMHC ligands (non-triggering) is sharply defined and close to the affinity threshold for foreign PMHC (triggering). Consequently, every TCR is likely to bind a subset of the ever-present self pMHC molecules with affinities differing only slightly from genuine foreign agonist PMHC. If TCR triggering and signaling were simply proportional to TCR occupancy by ligand, then the abundance of self pMHC could easily overwhelm genuine agonist signals, rendering this as an 
eLife digest The immune system identifies and combats foreign objects, including pathogens, in the body. T cells are key components of the immune system, and each has a unique variant of a signalling complex known as the T cell receptor on its surface. T cells scan the surfaces of other cells in search of antigens, which are peptides (fragments of proteins) that derive from foreign pathogens such as viruses. Successful recognition of a foreign peptide leads to an immune response that, in most cases, ultimately rids the body of the pathogen. Most importantly, however, the immune system must be able to discriminate between peptides that are produced naturally in the body ('self' peptides) and foreign or 'non-self' peptides. This is challenging because self peptides may have similar structures to non-self peptides and are often much more abundant.

Many models have been proposed to explain how T cells are able to detect just a few molecules of foreign peptide. According to some hypotheses the T cell receptors get together in clusters to function cooperatively; alternatively, it has been suggested that rapid binding of a foreign peptide to multiple T cell receptors sequentially can build up a strong signal. However, none of these phenomena have been directly observed.

O'Donoghue et al. now image the interactions between T cell receptors and peptides bound to molecules called major histocompatibility complexes (MHCs), and show that T cell activation can occur when a single foreign peptide binds to a single receptor. These interactions are longlived and ultimately result in the recruitment of ZAP70, which has an important role in the activation of T cells, to the complex formed by the T cell, the peptide and the MHC molecule. Therefore, any amplification of the activating signal transmitted by non-self peptides occurs following receptor binding, in contrast to previous models.

DOI: 10.7554/eLife.00778.002

ineffective discriminatory mechanism. Alternatively, each engagement of pMHC with TCR (serial triggering; Valitutti et alo, 1995), perhaps with some minimum engagement time (kinetic proof reading; McKeithan, 1995; Rabinowitz et al., 1996), might define the threshold for TCR triggering. Other proposed mechanisms elaborate further, suggesting (pseudo) heterodimers (Irvine et al., 2002; Krogsgaard et al., 2005) of self and agonist pMHC molecules or pMHC-independent forms of trans activation (Cooper and Qian, 2008) of multiple TCR by a single agonist pMHC may be at work. Proving or disproving any of these various possibilities based on current data is confounded by the vast difference between ensemble biochemical measurements and cell population behavior. Substantial ambiguity with respect to the actual molecular mechanisms responsible for antigen triggering of $T$ cells remains. A physically accurate understanding of this remarkable process will require simultaneous observations of the molecular binding kinetics, stoichiometry, and movement of individual signaling complexes in living T cells.

Here, we characterize the molecular interactions between PMHC and TCR, at the single molecule level, while simultaneously monitoring the local membrane recruitment of cytosolic Zeta-chain-associated protein kinase 70 (ZAP70) in live primary T cells. Every PMHC can be individually resolved and tracked for up to minutes before photobleaching by using a multi-timescale single molecule fluorescence imaging approach. Key to this strategy is the variable control of excitation light dose and exposure time to achieve hardware discrimination of molecular species with different mobilities. The pMHC molecules fall into two unambiguously distinguished classes: one undergoing fast random motion and the second moving slowly along linear trajectories. These slow moving PMHC are the bound PMHC:TCR complexes; they are only observed with agonist peptide and they spatially correlate with both TCR and recruitment of ZAP70. The linear trajectories of the PMHC:TCR complexes match the well-characterized cytoskeleton-driven movement of TCR during the formation of the immunological synapse (Campi et alo, 2005; Yokosuka et al., 2005; DeMond et al., 2008; Yu et al., 2012). Thus a lone agonist pMHC bound to TCR leads to stable engagement of the resulting complex with the cytoskeleton. Single molecule intensity calibration of the number of ZAP70 recruited to the vicinity of each agonist PMHC indicates that TCR are triggered in a 1:1 stoichiometry with $\mathrm{PMHC}$.

Associations of $\mathrm{pMHC}$ with TCR exhibited molecular binding dwell times with mean durations of $53.8 \pm 12.2 \mathrm{~s}$ and $5.2 \pm 0.2 \mathrm{~s}$ for AND and $5 \mathrm{c} . \mathrm{c7}$ TCRs, respectively. Individual dwell times are roughly exponentially distributed and are in general agreement with bulk solution measurements of pMHC:TCR 
kinetic off-rates for both TCRs (Corse et al., 2010; Huppa et al., 2010; Newell et al., 2011). However, dwell times measured from tracking experiments specifically correspond to spatial entrapment of pMHC with a TCR, or cluster of TCRs (Schamel and Alarcon, 2013), on the T cell surface. They do not necessarily correspond to individual molecular binding events with a single TCR. Indeed, recent studies (e.g., by FRET) have suggested that PMHC:TCR kinetic off-rates may be accelerated in living cells relative to in vitro measurements, possibly as a result of actively applied forces from the cytoskeleton (Huang et al., 2010; Huppa et al., 2010; Zhu et al., 2013). We explore the possibility that individual dwell times observed by tracking experiments could be composed of rapid unbinding and serial rebinding of pMHC to multiple TCR within a cluster. Results from a stochastic reaction-diffusion analysis, covering a wide range of parameter space, indicate that serial rebinding alone is unlikely to sustain entrapment. If pMHC thoroughly disengages from TCR, it will most likely escape. Structural flexibility within the pMHC:TCR complex (Adams et al., 2011; Hawse et al., 2012; Reboul et al., 2012) could give rise to apparently fast kinetics (e.g., in FRET measurements) without actual unbinding of pMHC from TCR. The tracking observations reported here directly reveal that the functional interaction between agonist PMHC and TCR is long-lived in living cells and that triggered TCR remain localized with the same $\mathrm{pMHC}$.

\section{Results}

\section{Characterization of agonist PMHC:TCR complexes}

We probe agonist PMHC:TCR complex dynamics in hybrid live cell-supported membrane junctions (Grakoui et al., 1999) (Figure 1A). The supported membrane is functionalized with MHC (IEk) and intercellular adhesion molecule-1 fusion with a blue fluorescent protein (ICAM1-TagBFP), both linked to the membrane via C-terminal poly-His tag binding to Ni-chelating lipids (Nye and Groves, 2008). The MHC is loaded with peptide (moth cytochrome c [MCC] agonist or null), which is covalently coupled in a 1:1 stoichiometry (verified by HPLC) to the photostable fluorophores Atto647N or Atto488 using maleimide-thiol chemistry. Upon contact between the T cell and the supported membrane, Leukocyte function-associated antigen 1 (LFA1)-ICAM1 binding leads to rapid cell spreading and formation of an essentially planar interface between the T cell and supported membrane, within which pMHC:TCR interactions occur.

At fast exposure times $(17.5 \mathrm{~ms})$ and high excitation powers $\left(0.2 \mathrm{~W} / \mathrm{cm}^{2}\right)$, all pMHC molecules are readily resolved (Figure 1B, left panel). The pMHC move as individual molecules, as identified by singlestep photobleaching, and can be continuously tracked for up to hundreds of frames (Figure 1C). In regions without a cell, pMHC exclusively exhibit random diffusive motion. The step-size distribution from these trajectories corresponds to a single lateral diffusion coefficient of 0.44 (SEM $=0.002) \mu \mathrm{m}^{2} / \mathrm{s}$, which is typical for supported membranes (Lin et al., 2010). Within the T cell junction, the step-size distribution becomes bimodal (Figure 1D). In addition to a fast component from freely diffusing PMHC, a distinct slow-moving component also appears.

At long exposure times $(500 \mathrm{~ms})$ and low excitation powers $\left(0.02 \mathrm{~W} / \mathrm{cm}^{2}\right)$, the fast moving $\mathrm{pMHC}$ fraction in Figure 1D is averaged over several pixels to form a relatively homogenous background. The slow moving molecules remain highly localized and can be unambiguously tracked for longer than a minute, using 1-10 s time-lapses (Figure 1B, right panel; Video 1). The slow moving PMHC molecules colocalize with TCR (Figure 2) and move in linear trajectories toward the geometric center of the live cell-supported membrane junction; these are the PMHC:TCR complexes. When MHC is loaded with a mixture of agonist and null peptides, with different fluorescent labels, only the agonist peptides are observed in the slow-moving complexes (Figure 3). The result is identical when the fluorophores are reversed, excluding the possibility that fluorophore effects could be responsible for binding.

The agonist $\mathrm{pMHC}$ densities used in these experiments range from 0.2 to 100 molecules $/ \mu \mathrm{m}^{2}$. The lowest densities are near thresholds for triggering $\mathrm{Ca}^{2+}$ flux (Manz et al., 2011) and below levels where stable TCR microclusters are readily visible (Figure 2 ), whereas the higher densities are well above the densities at which microclusters are observed (Campi et al., 2005; Yokosuka et alo, 2005; DeMond et al., 2008; Yu et al., 2012). Nonetheless, the observed single molecule pMHC motion at all densities is reminiscent of the well-characterized actomyosin-driven TCR microcluster radial transport in cells that are activated (Campi et al., 2005; Yokosuka et al., 2005; Kaizuka et al., 2007; DeMond et al., 2008; Yu et al., 2012). 


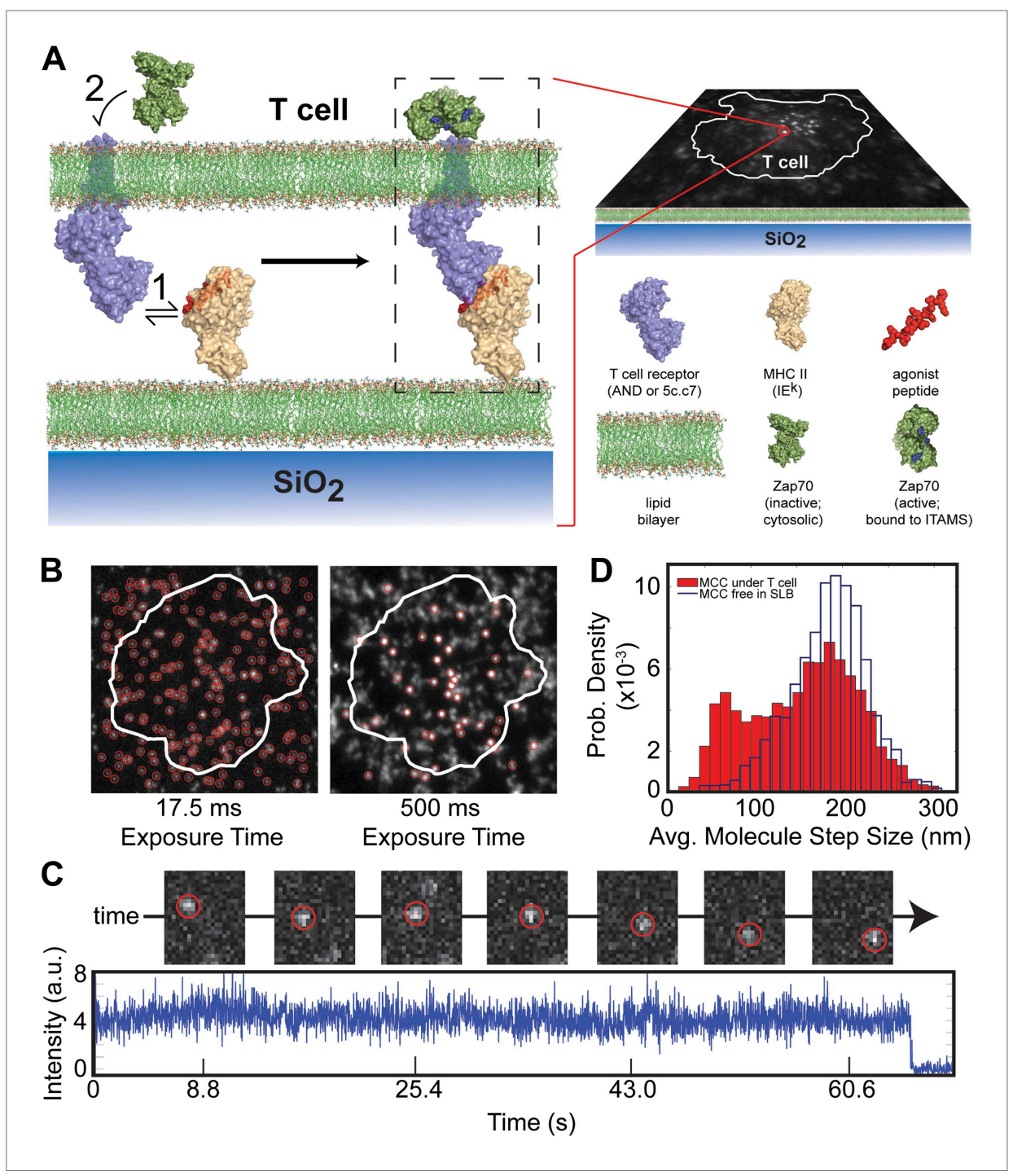

Figure 1. Agonist pMHC binding to TCR in T cells is revealed by changes in mobility. (A) Schematic of the hybrid live cell-SLB system. Binding of agonist PMHC to TCR (PDB, 3QIU) leads to phosphorylation of ITAMs, on the intracellular domain of the TCR, which is followed by recruitment of the kinase ZAP70 (PDB, 2OQI, 2OZO). We directly observe both PMHC:TCR binding and ZAP70:ITAM recruitment using single molecule fluorescence microscopy (bilayer adapted from Domanski et al., 2010). (B) At short exposure times (17.5 ms, left) all agonist pMHC molecules are readily resolved. Imaging with a long exposure time (500 ms, right) allows for unambiguous discrimination between the slow, TCR-bound fraction of agonist PMHC and the fast diffusing fraction. This also allows for long (1-10 s interval) time-lapse sequences (Video 1). Automated detection of single molecule features (red circles) is discussed in methods. (C) Representative intensity trace showing a single agonist pMHC molecule, identified by step photobleaching, bound continuously for $\sim 60$ s. (D) Step size histogram of single agonist pMHC molecules in a SLB is bimodal under T cells (red) and unimodal before addition of T cells (blue). pMHC molecules in (B)-(D) were labeled with Atto647N on the MCC peptide.

DOI: 10.7554/eLife.00778.003

\section{TCR triggering monitored by ZAP70 recruitment}

Two-color single molecule tracking is used to quantitatively monitor membrane recruitment of cytosolic ZAP70-EGFP (incorporated by retroviral transfection) to the locations of the PMHC:TCR complexes. Using a dual-view system in which chromatic aberrations have been mapped (Figure 4-figure supplement 1), spatial colocalization between the two channels to less than $105 \mathrm{~nm}$ is achieved. Immediately after cell landing, ZAP70 localizes to and moves together with the PMHC:TCR complexes (Figure 4A; Videos 2 and 3). For each frame in a tracking sequence, fluorescence intensity 


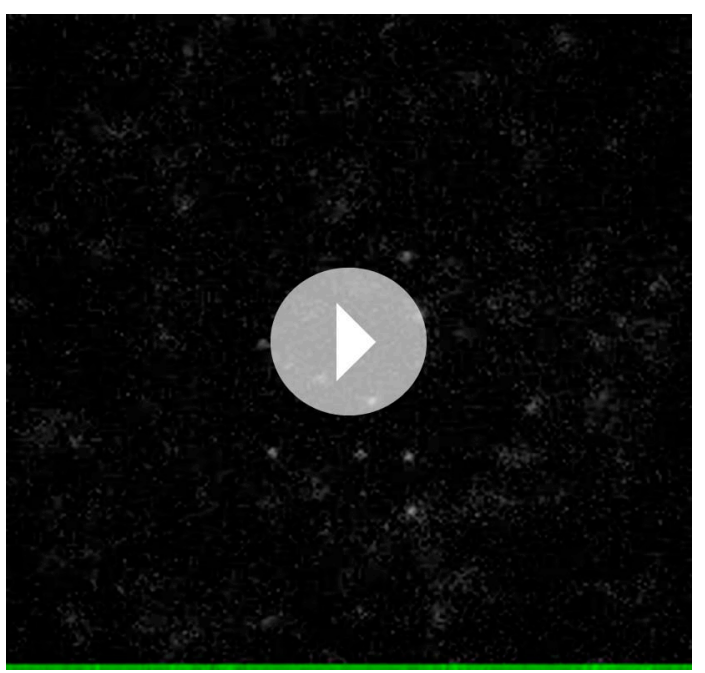

Video 1. A 3 s time-lapse video (189 frames) of agonist pMHC interacting with live 5c.c7 T cells (labeled with MCC-Atto488). A long (500 ms) exposure time allows for unambiguous discrimination between TCR-bound and unbound agonist $\mathrm{PMHC}$ in the bilayer. The hardware filtering approach utilized here facilitates particle detection in a relatively dense field of fluorophores, allowing for linking particles between frames even when long (1-10s) time lapses are introduced. This technique also has the advantage of using only one probe, compared to other techniques for detecting ligand binding, such as smFRET, which require two different color probes to detect one molecular binding event. These advantages allow for simultaneous two color single molecule tracking and kinetics measurements. DOI: 10.7554/eLife.00778.004

$(136.0$ counts \pm 0.04 SEM, integrated over a $315 \mathrm{~nm}$ square area for each feature) for the in Figure 4D corresponds to $2.9 \pm 0.04$ SEM ZAP70-EGFP per feature, given that the single molecule intensity is $47 \pm 2$ counts (SEM; $n=6$ ) in that cell. Each feature therefore contains on average $\sim 6$ ZAP70 molecules, since ZAP70-EGFP was expressed in a roughly 1:1 ratio with endogenous ZAP70 (selected by FACS and confirmed by western blotting) in these experiments. Each TCR complex has 10 ITAM domains that, when phosphorylated, can bind one ZAP70 each (Weiss and Littman, 1994). Thus observation of $~ 6$ ZAP70 per agonist PMHC suggests only a single TCR is triggered.

Brighter ZAP70-EGFP features tend to be located towards the geometric center of the cell at later time points (>5-10 min after T cell landing). These can be tracked for tens to hundreds of seconds, indicating that recruited ZAP70-EGFP remain stably associated with the PMHC:TCR complex while traveling along the same linear trajectories (Figure 4A; Videos 2 and 3). The observation of a range of ZAP70-EGFP stoichiometries (from 1 to 10 ZAP70-EGFP per pMHC:TCR complex) implies that some time-dependent accumulation of ZAP70 is likely to occur, although we have not definitively observed ZAP70-EGFP accumulation over time within individual intensity traces. Taken together, these observations demonstrate that engagement of TCR with an individual agonist pMHC molecule leads to stable association with the actin cytoskeleton, one-to-one TCR triggering (ITAM phosphorylation), and subsequent ZAP70 recruitment. Moreover, since every PMHC:TCR:ZAP70 complex is individually resolved in these experiments, we demonstrate that a single PMHC:TCR complex can lead to TCR triggering without molecular-scale association with other MHC molecules.

\section{Single molecule agonist PMHC:TCR binding kinetics}

Since the slow-moving $\mathrm{PMHC}$ can be clearly resolved from the fast moving component, the lifetime of molecules in this bound state is directly observable. The minimum detectable lifetime is limited by the 


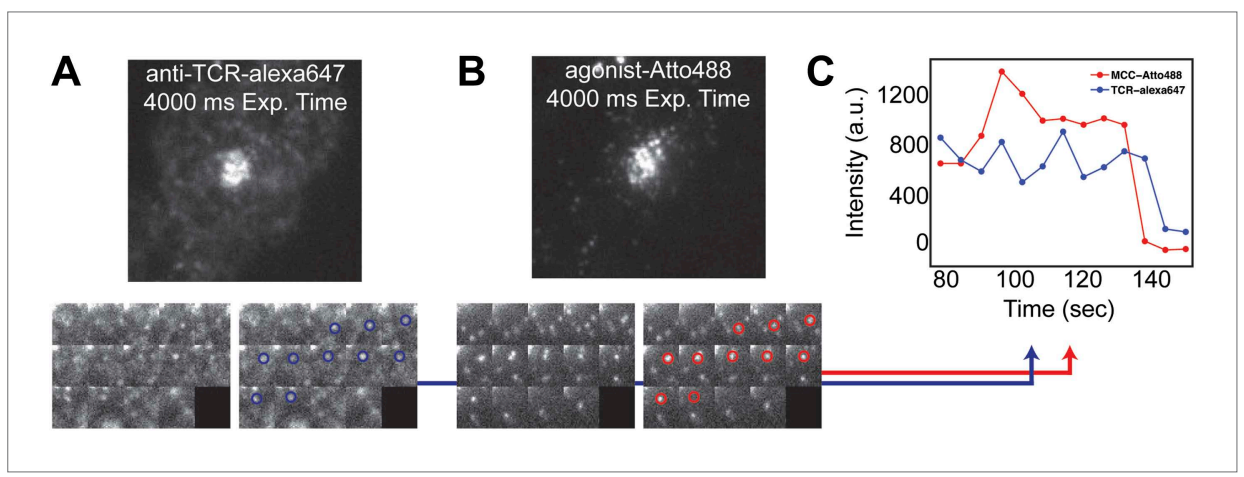

Figure 2. TCR and MCC agonist $\mathrm{pMHC}$ colocalization. (A) and (B) TCR and agonist $\mathrm{PMHC}$ colocalize in bulk in the central supramolecular assembly cluster (CSMAC) and (C) at the single molecule level. Although TCR clusters were not readily observed in these experiments (even using a $4 \mathrm{~s}$ camera integration time), previous reports indicate that TCR does cluster at the agonist density $\left(\sim 0.2\right.$ molecules/ $\left.\mu \mathrm{m}^{2}\right)$ used here (Varma et al., 2006). However, these reports used GPI-MHC, which is problematic because this GPI-linked protein is associated with clustering in supported membranes (Manz et al., 2011; Dustin and Groves, 2012). The $\mathrm{Ni}^{2+}$-chelating lipids used in supported membranes for the experiments reported here have been shown to increase the likelihood that attached proteins are monodispersed (Manz et al., 2011; Xu et al., 2011), and this is confirmed by direct single molecule observation in our experiments.

DOI: 10.7554/eLife.00778.005

fastest frame rate ( $17.5 \mathrm{~ms}$ per frame) and the maximum measureable lifetime is limited by photobleaching. For these single molecule tracking experiments, the temporal dynamic range spans from $\sim 50 \mathrm{~ms}$ to $\sim 5 \mathrm{~min}$. Unbinding and photobleaching are indistinguishable in fluorescence methods such as this. For molecular binding, characterized by a constant kinetic off-rate, the distribution of observed dwell times, $T_{\text {obs }}$ is described by

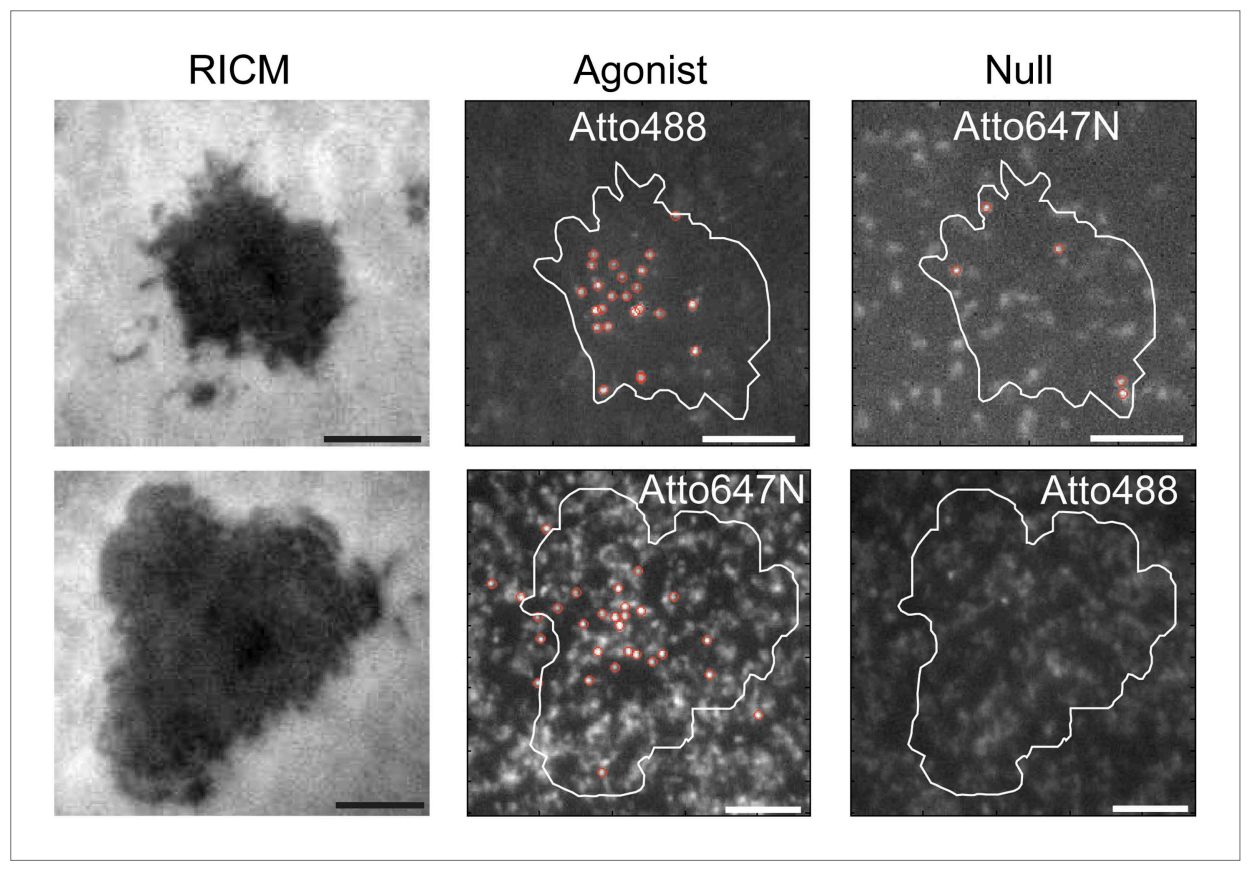

Figure 3. Agonist binding is specific and independent of fluorophore. RICM images map the footprint of T cell adhesion to the SLB (mediated through LFA1:ICAM1 binding). T cells engage SLBs conjugated with mixtures of independently labeled MCC agonist and null peptide MHC. Only the MCC agonist PMHC is observed in the slow moving fraction, irrespective of which fluorescent label (Atto488 or Atto647N) it carries.

DOI: 10.7554/eLife.00778.006 


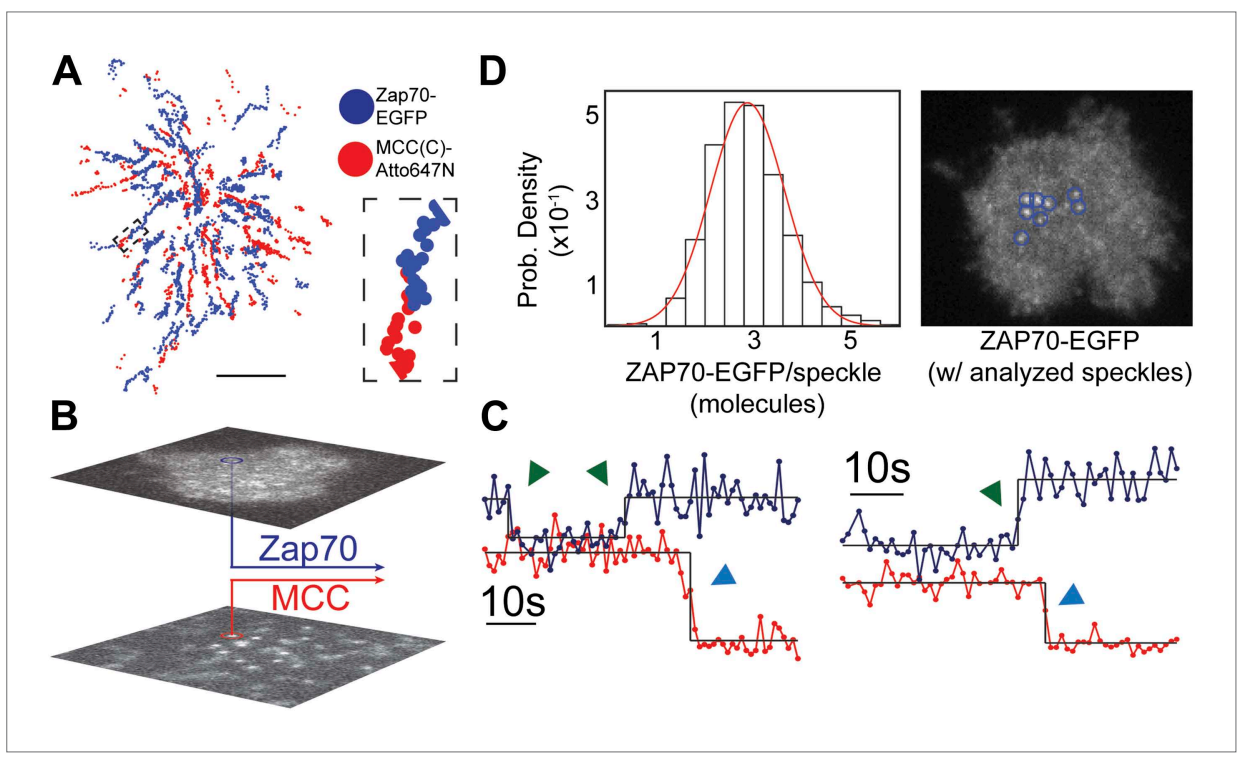

Figure 4. ZAP70 recruitment, stoichiometry, and movement are consistent with 1:1 agonist PMHC:TCR stoichiometry. (A) A spatial map of MCC-Atto647N single molecule (red) and ZAP70-EGFP (blue) puncta. Raw data are included as Videos 2 and 3. Data were recorded at 1 frame/s, such that each adjacent blue or red dot was recorded $1 \mathrm{~s}$ apart. Both single MCC agonist PMHC molecules and ZAP70-EGFP puncta follow linear trajectories towards the geometric center of the 2D cell-supported bilayer interface. (B) Single ZAP70-EGFP molecules recruited to single agonist $\mathrm{pMHC}$ molecules (labeled with MCC-Atto647N) are recorded using sub-pixel color registration (Figure 4-figure supplement 1). (C) Representative single molecule ZAP70-EGFP (blue) and MCC-Atto647N agonist pMHC (red) localized fluorescence intensity traces. Change-points are detected using a Bayesian change point algorithm (in black; see methods). Step decreases in MCC intensity (red) are most likely agonist pMHC:TCR unbinding events, since $T_{\text {off }} \ll T_{b}$. Step increases in ZAP70 intensity (blue) are attributed to ZAP70:ITAM binding. (D) ZAP70-EGFP puncta brightness histogram is symmetric and centered at 136.0 counts $\pm 0.04 \mathrm{SEM}$ and corresponds to an average of $2.9 \pm 0.04$ SEM EGFP molecules (using six single molecule ZAP70-EGFP traces from the same cell for intensity calibration). Bright ZAP70-EGFP speckles are detected from the raw data using an automated algorithm (blue circles; see methods). Scale bar $5 \mu \mathrm{m}$.

DOI: $10.7554 /$ eLife.00778.007

The following figure supplements are available for figure 4:

Figure supplement 1. Dual View color registration.

DOI: 10.7554/eLife.00778.008

Figure supplement 2. Single molecule ZAP70-EGFP.

DOI: 10.7554/eLife.00778.009

$$
f\left(T_{\text {obs }}\right)=\left(\left\langle T_{b l}\right\rangle^{-1}+\left\langle T_{\text {off }}\right\rangle^{-1}\right) e^{-T_{\text {obs }}\left(\left\langle\tau_{b l}\right\rangle^{-1}+\left\langle T_{\text {off }}\right\rangle^{-1}\right)}
$$

where $\left\langle T_{b l}\right\rangle^{-1}$ is the photobleaching rate $\left(k_{b}\right),\left\langle T_{\text {off }}\right\rangle^{-1}$ is the unbinding rate $\left(k_{\text {off }}\right)$, and $\left(\left\langle T_{b l}\right\rangle^{-1}+\left\langle T_{\text {off }}\right\rangle^{-1}\right)^{-1}=\left\langle T_{\text {obs }}\right\rangle$ is the observed mean dwell time in this experiment. The observed dwell time distributions are roughly exponential, as is expected for molecular binding. Thus by measuring both $k_{b l}$ and $\left\langle\tau_{\text {obs }}\right\rangle$ it is possible to determine $\left\langle\tau_{\text {off }}\right\rangle$ as long as $\left\langle\tau_{\text {obs }}\right\rangle \leq\left\langle\tau_{b l}\right\rangle$. We determine $\left\langle\tau_{\text {off }}\right\rangle$ to be $53.8 \pm 12.2$ $\mathrm{s}$ for AND and $5.2 \pm 0.2 \mathrm{~s}$ for $5 \mathrm{c} . \mathrm{c} 7$ TCRs for Atto488-labeled peptide agonist with $\left\langle T_{b l}\right\rangle$ of 300 and 30 $s$ respectively (Figure $5 A, B$ ). While fluorescent labels can affect binding kinetics, we measure similar values of $\left\langle\tau_{\text {off }}\right\rangle$ with both Atto647N and Atto488 labeled peptides (see, e.g., Figures 1C, 3, and 4A,C). $\left\langle T_{\text {off }}\right\rangle$ is also relatively unchanged at high agonist $\mathrm{pMHC}$ density $\left(100 \mathrm{molecules} / \mu \mathrm{m}^{2}\right)$, which is far above minimal levels required for T cell activation and observation of stable TCR microclusters (Manz et al., 2011) (Figure 5C). We observe that cytoskeleton disruption by the actin-binding molecule, Latrunculin A, moderately increases $\left\langle\tau_{\text {off }}\right\rangle$ with the AND TCR and had no significant effect on 5c.c7 kinetics (Figure 5C). Similarly, the dwell time distribution was only modestly affected by anti-CD4 (data not shown); however, the total number of TCR:pMHC complexes per cell was smaller in the anti-CD4 experiments, suggesting that the antibody interfered with pMHC:TCR binding. 


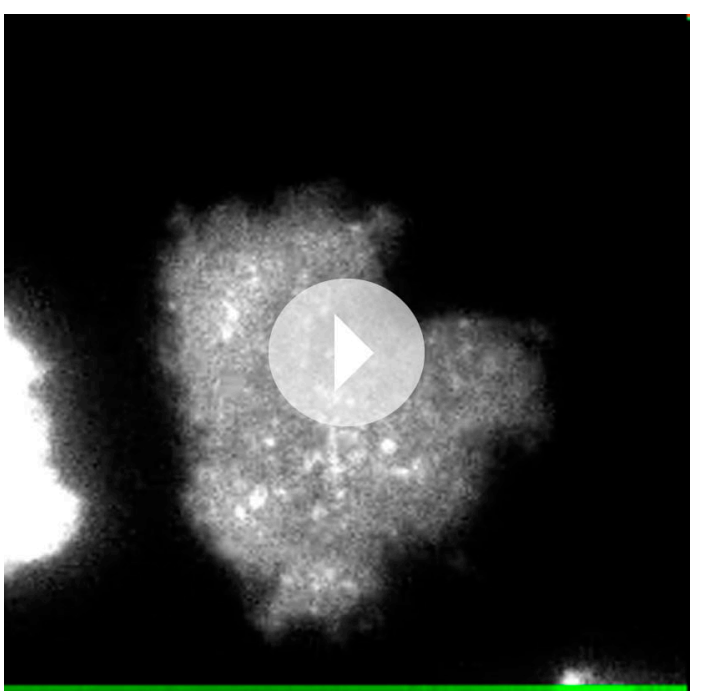

Video 2. Simultaneous observation of ZAP70-EGFP recruitment and $\mathrm{PMHC}$ :TCR binding immediately after a living AND T cell lands on the SLB. ZAP70-EGFP membrane recruitment (Video 2 ) and PMHC:TCR binding (Video 3) occur almost immediately after landing. Radial transport of pMHC:TCR:ZAP70 complexes commences immediately after landing. Data were recorded at 1 frame per second with a 500 ms integration time. These data were analyzed to create the spatial map of pMHC and ZAP70 positions displayed in Figure 4A. DOI: 10.7554/eLife.00778.010

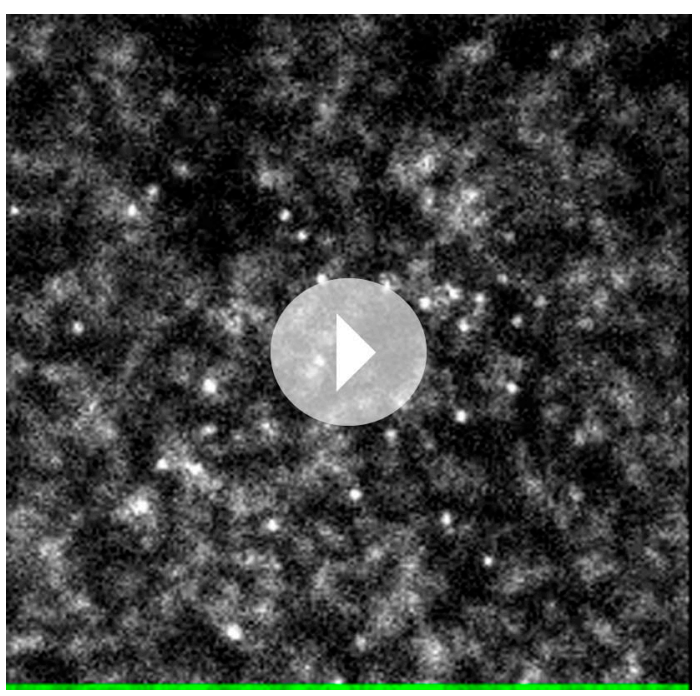

Video 3. Simultaneous observation of ZAP70-EGFP recruitment and $\mathrm{PMHC}$ :TCR binding immediately after a living AND T cell lands on the SLB. This video shows binding of PMHC:TCR, and is from the same cell as the ZAP70-EGFP data in Video 2. Agonist pMHC is labeled as MCC-Atto647N. These data were analyzed to create the spatial map of $\mathrm{pMHC}$ and ZAP70 positions displayed in Figure 4A.

DOI: 10.7554/eLife.00778.011
Tracking observations reveal the time intervals over which individual agonist $\mathrm{pMHC}$ molecules remain physically trapped within the immediate vicinity of the same TCR. Thus, although our measured dwell times in live cells are in general agreement with bulk solution measurements of pMHC:TCR kinetic off-rates for both TCRs (Figure 5C) (Corse et al., 2010; Newell et al., 2011), this may not result from the same reasons in each case. Unbinding of $\mathrm{pMHC}$ from TCR followed by rapid rebinding to the same TCR or another TCR within the same signaling cluster could conceivably lead to entrapment of pMHC for timescales longer than the lifetime of the molecular interaction. This has been hypothesized as a potential mode by which a small number of agonist pMHC could trigger a larger number of TCR (Govern et al., 2010; Huppa et al., 2010). Furthermore, recently reported single molecule measurements of pMHC:TCR binding kinetics in live cells, by force probe and by FRET, have suggested accelerated kinetic-off rates (Huang et al., 2010; Huppa et alo, 2010). We investigate this further below.

\section{Stochastic reaction-diffusion simulations}

We quantitatively assess the possibility of serial rebinding of agonist $\mathrm{PMHC}$ to multiple TCR within a TCR cluster using a stochastic reaction-diffusion simulation over a large range of $\left\langle T_{\text {off }}\right\rangle$ and TCR cluster size. The total time to escape for an individual molecule, which is the parameter directly measured in $\mathrm{PMHC}$ tracking experiments, is given by:

$$
T_{\text {esc }}=\sum_{i=0}^{n} T_{\text {off }}^{i}+\sum_{i=1}^{n} T_{\text {on }}^{i}+T_{\text {exit }}
$$

In this representation, $\tau_{\text {off }}^{i}$ and $\tau_{\text {on }}^{i}$ are the individual dwell times in the bound and unbound configurations, $n$ is the number of rebinding events, and $T_{\text {exit }}$ is the duration of the final unbound period prior to ultimate escape. For the stochastic simulation, $T_{\text {off }}^{i}$ and $T_{\text {on }}^{i}$ are treated as random variables with exponential distributions defined by the in situ measured values of $k_{\text {off }}$ and $k_{\text {on }}$ for pMHC:TCR binding, respectively. If the pMHC diffuses out of the TCR cluster prior to rebinding, it has escaped. Otherwise, the PMHC rebinds and the cycle repeats. Using the fastest $k_{\text {on }}$ $\left(0.17 \mu^{2} \mathrm{~s}^{-1} \mathrm{molecule}^{-1}\right)$ observed in similar hybrid live cell-SLB systems (Huppa et al., 2010) and the measured diffusion coefficient of $\mathrm{pMHC}$ in our supported membranes, we find that $T_{\text {esc }} \approx T_{\text {off }}$ for TCR clusters of the sizes observed experimentally (Varma et al., 2006) ( $\leq 100$ TCR molecules) (Figure 6). Only for unrealistically large TCR 


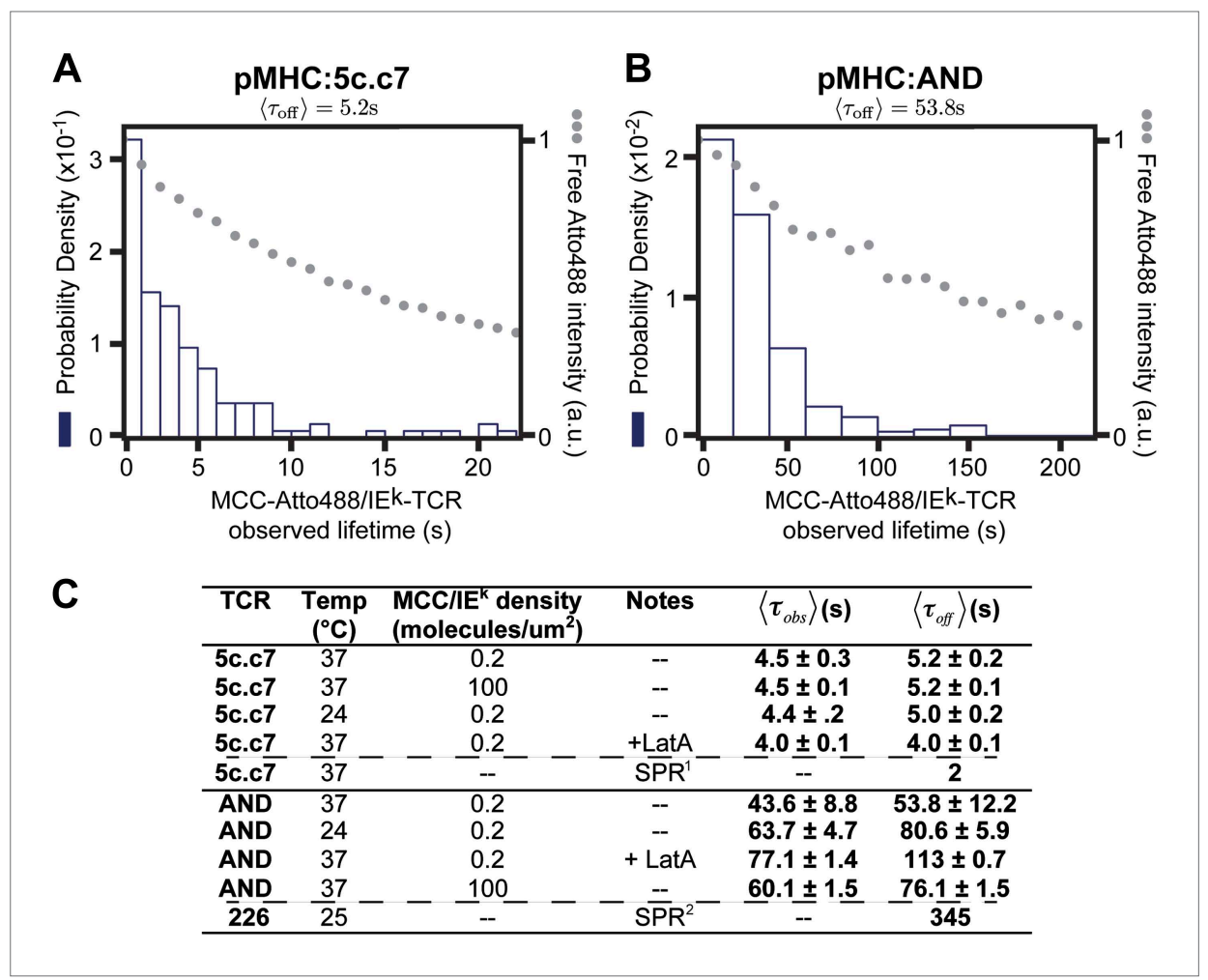

Figure 5. The distribution of live cell single molecule agonist PMHC:TCR molecular binding dwell times is observed directly. Measured dwell time distributions for both the 5c.c7 (A) and AND (B) TCRs are roughly exponential and match reported solution measurements. Bleaching times, $k_{b l}^{-1}$, (grey circles) are measured using agonist PMHC SLB standards without cells and with the same fluorescent label (Atto488) and are significantly longer than observed dwell times, $T_{\text {obs, }}$ for both TCRs. (C) Measured values for 5 c.c7 and AND CD4+ T cells under varying conditions. Values in columns five and six represent $\sim 300-600 \mathrm{MCC}$ agonist pMHC molecules per experimental condition from a population of 7-20 cells. Data are representative of at least 5 independent experiments performed on T cell blasts isolated from different mice for both the 5 c.c7 and AND TCRs. Uncertainty in the average across different mice, shown in columns five and six, is calculated as the standard error of the mean of the molecular averages from different mice. In some cases (e.g. for cytoskeleton disruption experiments with Latrunculin A) one experiment (representative of 7-10 cells and $100 \mathrm{~s}$ of single molecule measurements) may be performed, but these data are always compared to a control sample recorded on the same day with $T$ cell blasts from the same mouse. In these cases uncertainty is reported as the standard error of the mean of the molecular dwell time distribution. SPR measurements for 5 c.c $7{ }^{1}$ (Huppa et al., 2010) and AND-related 226 TCRs $^{2}$ (Newell et al., 2011), along with single molecule FRET measurements for $5 c . c 7{ }^{1}$ (Huppa et al., 2010), are shown for comparison.

DOI: 10.7554/eLife.00778.012

clusters ( 1000 TCR molecules) could rebinding within the same cluster lead to appreciable entrapment $\left(T_{\text {esc }}>T_{\text {exit }}\right)$ (Figure 6-figure supplement 1).

These simulations indicate that the observed values of $T_{\text {off }}$ are unlikely to be the result of rapid serial rebinding of one PMHC with many TCR within a TCR cluster. We note that although TCR clusters are not readily visible at the low antigen densities in these experiments, low level TCR clustering has been reported in the resting state by other methods (Schamel and Alarcon, 2013). Even with a very fast $k_{\text {on, }}$ if $\mathrm{pMHC}$ completely disengages from TCR for long enough to diffuse to an adjacent TCR, then the probability of complete escape from the TCR cluster is high. Only in the extreme limit, where $k_{\text {on }}$ is so fast that PMHC unbinding is predominantly followed by rebinding to the same TCR, is the escape time appreciably longer than the individual molecular dwell times. The distinction between rapidly rebinding the same TCR and a single engagement is largely semantic. However, it may reveal something about the mechanical stability of pMHC: TCR interactions, and could account for the apparently accelerated $k_{\text {off }}$ observed in other types of experiments. 


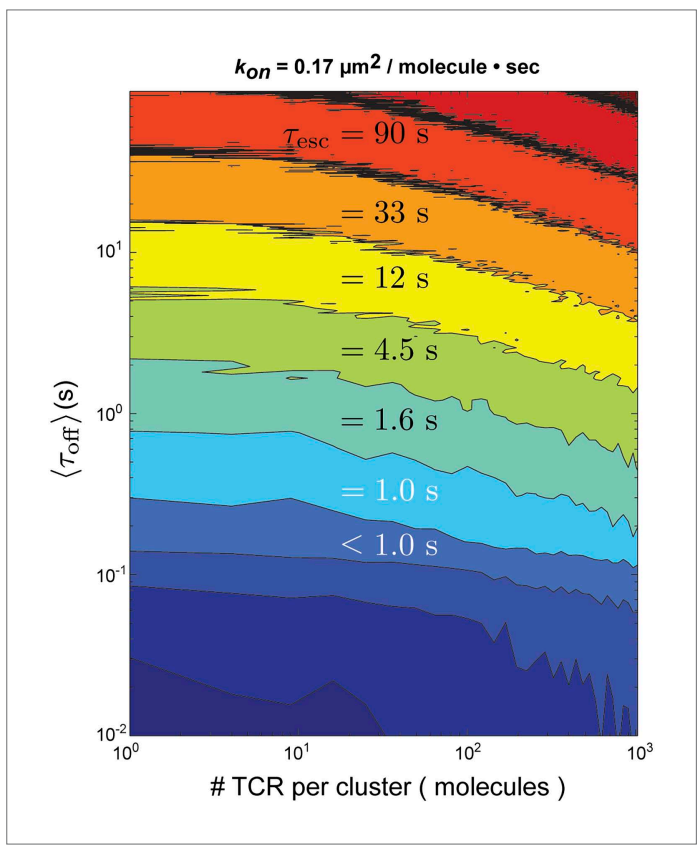

Figure 6. Stochastic reaction-diffusion simulation of time before MCC agonist PMHC escape from TCR clusters, $T_{\text {esc }}$ (in color; log scale), as a function of $T_{\text {off }}$ and TCR cluster size. For small TCR clusters (1-100 TCR molecules) $T_{\text {off }} \approx T_{\text {esa }}$ indicating no serial rebinding. Only for unrealistically large TCR clusters (1000-10,000 molecules) does $T_{\text {esc }}$ become appreciably longer than $T_{\text {off }}$. DOI: $10.7554 /$ eLife.00778.013

The following figure supplements are available for figure 6:

Figure supplement 1. Stochastic reaction-diffusion simulation of time before MCC agonist pMHC escape from TCR clusters.

DOI: $10.7554 /$ eLife.00778.014

\section{Discussion}

All evidence reported here suggests that individual agonist $\mathrm{PMHC}$ remain bound to the same TCR for at least several seconds for the 5c.c7 TCR and for approximately one minute for the AND TCR in live cells. Subsequent recruitment of ZAP70 indicates TCR are triggered and movement of the complexes along linear trajectories confirms stable association with the actin cytoskeleton. All of this occurs as a result of a lone agonist $\mathrm{PMHC}$ binding $\mathrm{TCR}$, without involvement of other $\mathrm{MHC}$. The measured average pMHC:TCR:ZAP70 stoichiometry indicates TCR triggering is most likely $1: 1$ with agonist $\mathrm{PMHC}$. Supernumerary triggering of multiple TCR by a single $\mathrm{PMHC}$ is not observed on the timescales of minutes investigated in these experiments. The original serial triggering model (Valitutti et al., 1995) drew its conclusions from the extent of TCR down regulation measured $5 \mathrm{hr}$ after exposure of $\mathrm{T}$ cells to antigen-pulsed APCs. Additionally, the lack of molecular-scale cooperativity in TCR triggering by agonist $\mathrm{pMHC}$ indicates that observed cooperativity at the level of cellular calcium response, at similar ligand densities and timescales (Manz et al., 2011), is most likely due to intracellular feedback mechanisms (Altan-Bonnet and Germain, 2005; Chan et al., 2004; Das et al., 2009; Stefanová et al., 2003).

The timescales of the pMHC:TCR interactions we observe in live cells are consistent with SPR measurements of $k_{\text {off }}$ for the 5c.c7 TCR and 226 TCR, which is nearly identical to AND (Figure $5 \mathrm{C}$ and Figure 7) (Corse et al., 2010; Newell et al., 2011). They are also consistent with reports of a $\sim 2 \mathrm{~s}$ kinetic threshold for thymic selection determined in vivo (Williams et al., 1999; Palmer and Naeher, 2009). Stochastic reaction-diffusion analysis of the measured kinetic and mobility parameters indicates that rapid serial rebinding of agonist PMHC to multiple TCRs in a signaling cluster is unlikely to be a universal mechanism for ligand discrimination and rapid signal amplification in $\mathrm{T}$ cells.

The intercellular geometry as well as active processes within the T cell have long been suspected to influence pMHC:TCR interactions (Shaw and Dustin, 1997; Qi et al., 2001; Burroughs and Wulfing, 2002; Zhu et al., 2013). Direct in situ measurements of individual pMHC:TCR binding kinetics, such as we report here, are extremely limited (Huang et al., 2010; Huppa et al., 2010; Axmann et al., 2012) but informative comparisons can be made. Notably, a recent intramolecular FRET study of the 5c.c7 TCR binding MCC pMHC reports short ( $\left.\left\langle T_{\text {off }}\right\rangle \sim 150 \mathrm{~ms}\right)$ in situ 2D dwell times, nearly 35 times faster than the $\left\langle\tau_{\text {off }}\right\rangle=5.2 \pm 0.2 \mathrm{~s}$ we measured by tracking. This same study measures $\left\langle T_{\text {off }}\right\rangle 25$ times longer under conditions of cytoskeleton disruption, essentially in agreement with tracking observations. This result has been interpreted to mean that the actin cytoskeleton actively destabilizes agonist pMHC:TCR complexes. In contrast, we observe very small effects of actin disruption on agonist pMHC dwell times for AND and essentially no effect with 5c.c7 TCR (Figure 5C).

It is conceivable that the agonist pMHC:TCR complex does not remain bound in the same structural configuration for the duration of engagement. If mechanical coupling to actin significantly reduces the apparent $\left\langle\tau_{\text {off }}\right\rangle$ in a single molecule FRET measurement, but not in a single molecule tracking experiment, this raises the possibility that mechanical forces can induce conformational alterations in agonist pMHC:TCR without complete disengagement of the complex. Recent structural studies of pMHC:TCR indicate the possibility of such flexibility (Adams et al., 2011; Hawse et al., 2012; Reboul et al., 2012). 


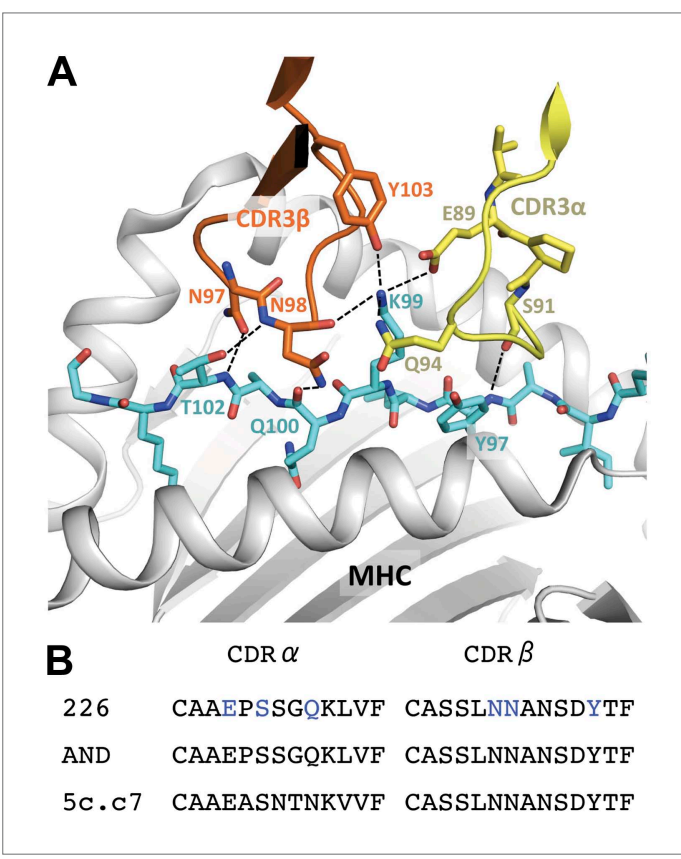

Figure 7. Interaction of the 226 TCR with the MCC peptide. (A) CDR3 $\alpha$ (yellow) and CDR3 $\beta$ (orange) loops form all the specific interactions with the peptide (cyan) Hydrogen bonds between the CDR3 loops and the $M C C$ residues are shown by dashed lines (PDB, 3QIU) (B) Comparison of CDR3 loops between the 226 and AND TCRs reveal identical sequences and suggest similar binding kinetics. 226 and AND also share $\mathrm{Va}, \mathrm{Ja}$, $V \beta$, and $J \beta$ gene segments that encode the residues specific for interaction with IEk (Newell et al., 2011). The residues involved in hydrogen bonds between 226 and $\mathrm{MCC}$ are shown in blue.

DOI: 10.7554/eLife.00778.015
While putative conformational changes would not affect single-molecule tracking measurements, they could produce a FRET signature (Ha et al,, 1999; Majumdar et al., 2007). Under such a scenario, apparently fast $\left\langle T_{\text {off }}\right\rangle$ observed by FRET imaging (Huppa et al., 2010) may not correspond to actual molecular unbinding and escape of agonist PMHC from TCR.

In the aggregate, the data reported here indicate that spatially discrete pMHC:TCR:ZAP70 complexes form according to molecular mass action laws with relatively predictable chemical kinetics and stoichiometry in living cells. The observed pMHC:TCR molecular binding kinetics mirror solution measurements and we observe no evidence for molecular scale cooperativity in the triggering of TCR by agonist PMHC (at low agonist density). Thus any amplification or other form of signal processing must occur downstream of TCR triggering.

\section{Materials and methods}

\section{DNA, protein, and $T$ cell preparation}

A plasmid containing enhanced green fluorescent protein fused to $C D 3$ zeta-chain-associated protein of $70 \mathrm{kDa}$ (Zap70-EGFP) was a gift of Takashi Saito, RIKEN Research Center for Allergy and Immunology, Yokohama, Japan (Yokosuka et alo, 2005). The Zap70-EGFP gene was amplified by $\mathrm{PCR}$ and subcloned into a murine stem cell virus parent vector (pMSCV).

Bi-hexahistidine-tagged major histocompatibility complex (MHC) class II I-Ek protein was produced and purified as previously described (Nye and Groves, 2008). A decahistidine-tagged ICAM1-TagBFP fusion protein was generated by PCR amplifying the TagBFP sequence (Evrogen Inc., Moscow, Russia) and subcloning it into a pN1ICAM1 vector. The entire Icam1-TagBFP gene was then further subcloned into the pFastBac1 vector (Invitrogen Inc., Carlsbad, CA), which was used to generate baculovirus for infection of High Five cells (Invitrogen Inc.). The ICAM1-TagBFP was subsequently purified on a Ni-NTA-agarose affinity column, eluted with an imidazole gradient, dialyzed, and stored in Tris buffer containing $10 \%$ glycerol. AND CD4+ T cells (Kaye et alo, 1989) and 5 c.c7 CD4+ T cells were harvested and cultured essentially as previously described (Smith et al., 2011; Smoligovets et al., 2012).

T cells were transduced with Zap70-EGFP and sorted using fluorescence-activated cell sorting (FACS) according to viability and EGFP expression. The population of transduced cells that was used expressed EGFP at no more than $50 \%$ of the highest EGFP level in the overall EGFP-positive population.

\section{Peptide purification and labeling}

Using the basic sequence of moth cytochrome c (amino acids 88-103) and previously described variants (Huppa et al., 2010), the following peptides were synthesized by David King at the HHMI Mass Spectrometry Laboratory at UC Berkeley and/or commercially (Elim Biopharmaceuticals, Hayward, CA): MCC (ANERADLIAYLKOATK), MCC(C) (ANERADLIAYLKQATKGGSC), MCC-null(C) (ANERAELIAYLTQAAKGGSC). For fluorophore labeling, cysteine-containing peptides were dissolved in a small amount of phosphate buffer and mixed in a 1:2 molar ratio with Atto $647 \mathrm{~N}$ resuspended in a small amount of 1-propanol or lyophilized Atto 488 (Atto-Tec GmbH, Siegen, Germany) and labeled using maleimide-thiol chemistry. The peptides were then incubated at room temperature for at least 
$1 \mathrm{hr}$ and purified on a C18 reverse phase column (Grace-Vydac, Deerfield, IL) and $\mathrm{H}_{2} \mathrm{O}$ :acetonitrile gradient using ÄKTA explorer 100 FPLC system (Amersham Pharmacia Biotech, Piscataway, NJ). Peptide identity was confirmed after purification using mass spectrometry.

\section{Microscopy}

TIRF experiments were performed on an inverted microscope (Nikon Eclipse Ti; Technical Instruments, Burlingame, CA) using a custom-built laser launch with $488 \mathrm{~nm}$ (Sapphire HP; Coherent Inc., Santa Clara, CA) and $640 \mathrm{~nm}$ (Cube; Coherent Inc.) diode lasers, as described previously (Smith et al., 2011). Laser powers measured at the sample were $0.8 \mathrm{~mW}(640 \mathrm{~nm})$ and $0.5 \mathrm{~mW}(488 \mathrm{~nm})$ for $500 \mathrm{~ms}$ exposures and $4.4 \mathrm{~mW}(640 \mathrm{~nm}$ ) for $17.5 \mathrm{~ms}$ exposures. A dichroic beamsplitter (z488/647rpc; Chroma Technology Corp., Bellows Falls, VT) reflected the laser light through the objective lens (Nikon 1.49 NA TIRF; Technical Instruments, Burlingame, CA) and fluorescence images were recorded using an EM-CCD (iXon 597DU; Andor Inc., South Windsor, CT) after passing through a laser-blocking filter (Z488/647 M; Chroma Technology Corp., Bellows Falls, VT). Bandpass filters (FF03 525/50; Semrock Inc., Rochester, NY and ET 700/75, Chroma Technology Corp., Bellows Falls, VT) were placed in a DualView 2 Simultaneous Imaging System (Photometrics, Tuscon, AZ). Colors were registered before every two-color experiment by imaging $100 \mathrm{~nm}$ Tetraspec beads (Invitrogen Inc.) deposited on a coverslip patterned with a $\mathrm{Cr}$ grid with $\sim 80 \mathrm{~nm}$ width and 3-4 $\mu \mathrm{m}$ pitch $\mathrm{Cr}$ lines, since the Tetraspec beads preferentially bind the regular $\mathrm{Cr}$ pattern. Exposure times and time-lapse periods for most experiments were set using image collection software (MetaMorph 7.5; Molecular Devices Inc., Downingtown, PA), which drives an external shutter (Uniblitz LS6; Vincent Associates, Rochester, NY). Exposure time and Fast Kinetics Mode for short $(17.5 \mathrm{~ms}$ ) integration time experiments were set using Andor Solis (Andor Inc., South Windsor, CT). Exposure times were measured directly from the Fire output of the EM-CCD using an oscilloscope (TDS 210; Tektronix, Inc., Beaverton, OR).

\section{Imaging chamber and supported lipid bilayer preparation}

Single unilamellar vesicles (SUVs) were formed by tip sonication of a solution composed of $98 \mathrm{~mol} \%$ 1,2-dioleoyl-sn-glycero-3-phosphocholine (DOPC) and 2 mol \% 1,2-dioleoyl-sn-glycero-3-[( $\mathrm{N}$-(5amino-1-carboxypentyl) iminodiacetic acid) succinyl] (nickel salt) (Ni ${ }^{2+}$-NTA-DOGS) (Avanti Polar Lipids, Alabaster, AL) in Mill-O water (EMD Millipore, Billerica, MA). Tip sonication was preferred to vesicle extrusion due to the introduction of significant levels of fluorescent impurities into the SUVs during extrusion. Prior to experiments, \#2 $40 \mathrm{~mm}$ diameter round coverslips were ultrasonicated for $30 \mathrm{~min}$ in 50:50 isopropyl alcohol:water, rinsed thoroughly in Milli-Q water (EMD Millipore, Billerica, MA), etched for $5 \mathrm{~min}$ in piranha solution (3:1 sulfuric acid:hydrogen peroxide), and again rinsed thoroughly in Milli-Q water. The coverslips were used in the assembly of FCS2 Closed Chamber Systems (flow cells; Bioptechs, Butler, PA), which were pre-filled with Tris-buffered saline (TBS; $19.98 \mathrm{mM}$ Tris, 136 $\mathrm{mM} \mathrm{NaCl}, \mathrm{pH}$ 7.4; Mediatech Inc., Herndon, VA). SUVs were then flowed into the chambers, and bilayers were allowed to form for at least $30 \mathrm{~min}$. The bilayers were rinsed once with TBS, incubated for 5 min with $100 \mathrm{mM} \mathrm{NiCl}$ in TBS, rinsed with TBS, and then rinsed with a T cell imaging buffer composed of $1 \mathrm{mM} \mathrm{CaCl}, 2 \mathrm{mM} \mathrm{MgCl} 2,20 \mathrm{mM}$ HEPES, $137 \mathrm{mM} \mathrm{NaCl}, 5 \mathrm{mM} \mathrm{KCl}, 0.7 \mathrm{mM} \mathrm{Na}_{2} \mathrm{HPO}_{4}$, $6 \mathrm{mM} \mathrm{d}$-glucose, and $1 \% \mathrm{wt} / \mathrm{vol}$ bovine serum albumin. $48 \mathrm{hr}$ prior to experiments, MHC was loaded with peptide at $37^{\circ} \mathrm{C}$ in a buffer composed of $1 \% \mathrm{wt} / \mathrm{vol}$ bovine serum albumin in phosphate-buffered saline and brought to $\mathrm{pH} 4.5$ with citric acid. Unbound peptide was separated from peptide loaded MHC (pMHC) using 10k spin concentrators (Amicon Ultra, Cork, Ireland) and then pMHC was diluted in imaging buffer. ICAM1-TagBFP and pMHC were further diluted with imaging buffer, introduced into the flow cells, and incubated for $35 \mathrm{~min}$ followed by a rinse with imaging buffer. $T$ cells resuspended in imaging buffer and added to the flow cells $35 \mathrm{~min}$ after the final rinse and imaged immediately for 30-60 min. To visualize TCR, T cells were incubated in a solution of $1 \mu \mathrm{l}$ Alexa 647 (Invitrogen Inc.)labeled $\mathrm{H} 57$ anti-TCR Fab and $100 \mu \mathrm{l}$ imaging buffer for $20 \mathrm{~min}$ at $4^{\circ} \mathrm{C}$ prior to the regular imaging buffer resuspension. All other incubations during this protocol were performed at room temperature, and imaging experiments were performed at $37^{\circ} \mathrm{C}$.

\section{Data analysis}

Single molecule diffraction-limited spots were detected in raw .tif image stacks of agonist pMHC labeled with MCC-Atto488 and MCC-647N molecules by filtering for both size and intensity and linked into tracks using published particle detection and tracking algorithms (Crocker and Grier, 1996) adapted for MATLAB (The Mathworks; Natick, MA) by Daniel Blair and Eric Dufresne 
(http://physics.georgetown.edu/matlab/; accessed 16 August 2012). Size and intensity thresholds were first determined by eye using a test data set and then applied uniformly to all data collected with the same exposure time and incident laser intensities. Single molecules were identified by step photobleaching detected in an automated way using a Bayesian change point detection algorithm (Ensign and Pande, 2009).

The brightness of ZAP70-EGFP features varies from a single molecule to several molecules, and different brightness features are detected using slightly different methods, despite the fact that the features are physically similar. Bright ZAP70-EGFP features (as shown in Figure 4A,D) were detected using the same algorithm as is used for single molecule $\mathrm{pMHC}$. The lower signal-to-noise single molecule ZAP70-EGFP intensity traces like those in Figure $4 C$ were obtained by summing the intensity of the ZAP70-EGFP channel using the agonist pMHC (labeled with Atto647N) molecule position as a mask, as is explained in the main text.

The lifetime of the bright ZAP70-EGFP speckles is difficult to accurately assess due to the fluctuating background and varying speckle intensity (which biases measurement towards brighter, longer-lived fluorescent features), but speckle lifetimes appear to be longer than the single molecule ZAP70-EGFP lifetimes. Single molecule ZAP70-EGFP molecules are uncorrected for photobleaching of both ZAP70EGFP and agonist pMHC and therefore the range of binding times reported (12-107 s) only serves as a lower bound for the molecular ZAP70 dwell time.

The agonist pMHC step size distribution at $17.5 \mathrm{~ms}$ resolution in Figure 1D is populated using similar particle detection and tracking methods to the 500 ms resolution analysis., Agonist pMHC:TCR binding kinetics cannot be uniquely inferred from the step size distribution, since the step size distribution is a time-independent analysis. For instance, the step size distribution measured over a certain time period with $2 k_{\text {on }}$ and $2 k_{\text {off }}$ would appear identical to a scenario with $k_{\text {on }}$ and $k_{\text {off }}$.

Lifetime distributions are roughly exponential and of the form $f\left(\tau_{\text {obs }}\right)=\left\langle\tau_{\text {obs }}\right\rangle^{-1} e^{-\tau_{\text {obs }} /\left\langle\tau_{\text {obs }}\right\rangle}$, where $T_{\text {obs }}$ is the observed dwell time in our experiments. The individual kinetic transitions were derived assuming the following model:

$$
p M H C_{\text {free }} \underset{k_{\text {off }}}{\stackrel{k_{\text {on }}}{\longrightarrow}} p M H C: T C R \stackrel{k_{b b}}{\longrightarrow} p M H C_{\text {bleached }}
$$

where $p M H C_{\text {free }}$ is the fast-mobility state, $p M H C: T C R$ is the slow-mobility state (or the TCR stably bound state), $\mathrm{pMHC} \mathrm{C}_{\text {bleached }}$ is the bleached slow-mobility state, $k_{\text {off }}$ and $k_{\text {on }}$ are the rates of transitions between the bound and the free $\mathrm{pMHC}$, and $k_{b l}$ is the rate of transition from bound pMHC:TCR to photobleached PMHC. We assume that transititions between states follow a Markov memory-less process and derive a probability density function, $f\left(\tau_{\text {obs }}\right)$, for the single molecule dwell time distribution: $f\left(\tau_{\text {obs }}\right)=\left(\left\langle\tau_{\text {bl }}\right\rangle^{-1}+\left\langle\tau_{\text {off }}\right\rangle^{-1}\right) e^{-\tau_{\text {obs }}\left(T_{b l}||^{-1}+\left\langle\left\langle_{\text {off }}\right\rangle^{-1}\right)\right.}$, where $\left(\left\langle T_{b \mid}\right\rangle^{-1}+\left\langle\tau_{\text {off }}\right\rangle^{-1}\right)^{-1}=\left\langle\tau_{\text {obs }}\right\rangle$ is the observed mean dwell time in our experiments. Agonist pMHC labeled with Atto488 and Atto647N SLB bleaching curves were background subtracted and then fit to an exponential decay function of the form $f(t)=k_{b l} e^{-k_{b l} t}$. Fitting was done using MATLAB.

\section{Stochastic kinetic simulation}

Simulations were performed using MATLAB. Our simulation models a TCR cluster as a square lattice upon which agonist PMHC molecules bind discrete TCR lattice sites for duration $T_{\text {off }}^{i}, T_{\text {off }}^{i}$ where is treated as a random variable drawn from an exponential distribution with mean equal to $\left\langle\tau_{\text {off }}\right\rangle \cdot\left\langle\tau_{\text {off }}\right\rangle$ is varied over several orders of magnitude and is chosen to match measured values from the literature. pMHC are initially placed at a randomly lattice position drawn from a uniform distribution. After each time period (determined by $T_{\text {off }}^{i}$ ), the agonist $\mathrm{pMHC}$ molecule steps to a new lattice site or stays at the same lattice site (the lattice spacing is set to $10 \mathrm{~nm}$ to roughly follow the size of the TCR complex [Newell et al., 2011; Yin et al., 2012]) until the agonist PMHC is no longer on the TCR cluster, such that $T_{\text {esc }}^{k}=\sum_{i=0}^{n} T_{\text {off }}^{i}+\sum_{i=1}^{n} T_{\text {on }}^{i}+T_{\text {exit }}$ where $n$ indicates the number of steps an individual agonist pMHC molecule takes before exiting the TCR cluster, $T_{\text {on }}$ is the time period between unbinding and binding events, and $\tau_{\text {exit }}$ is the time between the last unbinding event and the ultimate exit from the TCR cluster. Step size is treated as a combination of two independent random variables, $\left(\Delta x, \Delta y_{1}\right)$ drawn from Gaussian distributions with mean 0 and standard deviation $\sqrt{2 D_{S L B} T_{\text {on }}}$. The step size is then a random variable $\Delta r=\sqrt{\Delta x^{2}+\Delta y^{2}}$ and the angle of displacement is drawn from a uniform distribution. 
The interval between binding events, $T_{\text {on }}$ is treated as a random variable drawn from an exponential distribution with mean $k_{\text {on }} \rho_{\text {TCR }}$ where the density of TCR, $\rho_{T C R}$, is taken to be 10,000 molecules $/ \mu m^{2}$ (as in the central supramolecular activation cluster). In this way $\left\langle T_{\text {off }}\right\rangle=\frac{1}{k} \sum_{k} T_{\text {esc }}^{k}$, where $k$ is the number of iterations (100 in the case of Figure 6 and Figure 6 -figure supplement 1), is calculated for every combination of $\left\langle T_{\text {off }}\right\rangle$, TCR cluster size, and $k_{\text {on }}$. Note that since $T_{\text {off }} \gg T_{\text {on' }} \frac{T_{\text {esc }}}{T_{\text {off }}} \approx\langle n\rangle$, where $\langle n\rangle$ is equivalent to the TCR cluster size. This relationship between $\frac{T_{\text {esc }}}{T_{\text {of }}}$ and TCR cluster size can be seen in Figure 6$T_{\text {off }}$

figure supplement 1. It is possible that agonist pMHC binding interactions with CD4 could slow the mobility of an individual agonist $\mathrm{PMHC}$ within a TCR cluster relative to $D_{S L B}$ when the agonist $\mathrm{PMHC}$ are unbound from TCR. This could hypothetically lead to entrapment and long single molecule tracks (like those reported here) in the absence of direct, sustained agonist pMHC-TCR interactions. While such a mechanism is conceivable, there is no direct evidence for such a tethering mechanism in the literature.

\section{Acknowledgements}

The content is solely the responsibility of the authors and does not necessarily represent the official views of the National Institutes of Health. We thank David King of the HHMI Mass Spec Facility for peptide synthesis and Mass Spectroscopy, Nicole Fay, Kate Alfieri, and Niña Hartman for MHC purification, and Brian D Belardi for a critical reading of the manuscript.

\section{Additional information}

Funding

\begin{tabular}{lll} 
Funder & Grant reference number & Author \\
\hline National Institute of Allergy & PO1 Al091580 & Geoff P O'Donoghue, \\
and Infectious Diseases & & Rafal M Pielak, \\
& & Alexander A Smoligovets, \\
& Jenny J Lin, Jay T Groves \\
\hline
\end{tabular}

The funders had no role in study design, data collection and interpretation, or the decision to submit the work for publication.

Author contributions

GPO, RMP, Conception and design, Acquisition of data, Analysis and interpretation of data, Drafting or revising the article; AAS, JJL, Acquisition of data, Drafting or revising the article; JTG, Conception and design, Analysis and interpretation of data, Drafting or revising the article

Ethics

Animal experimentation: CD4+ T-cell blasts were cultured from the lymph nodes and spleens of first generation AND x B10.BR and 5c.c7 TCR transgenic mice on day 1 of the T-cell protocol in accordance with Lawrence Berkeley National Laboratory Animal Welfare and Research Committee-approved protocol 17702.

\section{References}

Adams JJ, Narayanan S, Liu B, Birnbaum ME, Kruse AC, Bowerman NA, et al. 2011. T cell receptor signaling is limited by docking geometry to peptide-major histocompatibility complex. Immunity 35:681-93. doi: 10.1016/j. immuni.2011.09.013.

Altan-Bonnet G, Germain RN. 2005. Modeling T Cell antigen discrimination based on feedback control of digital ERK responses. PLoS Biol 3:e356. doi: 10.1371/journal.pbio.0030356.

Axmann M, Huppa JB, Davis MM, Schütz GJ. 2012. Determination of interaction kinetics between the T cell receptor and peptide-loaded MHC class II via single-molecule diffusion measurements. Biophys J 103:L17-9. doi: 10.1016/j.bpj.2012.06.019.

Bunnell SC, Hong DI, Kardon JR, Yamazaki T, McGlade CJ, Barr VA, et al. 2002. T cell receptor ligation induces the formation of dynamically regulated signaling assemblies. J Cell Biol 158:1263-75. doi: 10.1083/jcb.200203043. Burroughs NJ, Wulfing C. 2002. Differential segregation in a cell-cell contact interface: the dynamics of the immunological synapse. Biophys J 83:1784-96. doi: 10.1016/S0006-3495(02)73944-1. 
Campi G, Varma R, Dustin ML. 2005. Actin and agonist MHC-peptide complex-dependent T cell receptor microclusters as scaffolds for signaling. J Exp Med 202:1031-6. doi: 10.1084/jem.20051182.

Chan C, Stark J, George AJ. 2004. Feedback control of T-cell receptor activation. Proc Biol Sci 271:931-9. doi: 10.1098/rspb.2003.2587.

Cooper JA, Qian H. 2008. A mechanism for Src kinase-dependent signaling by noncatalytic receptors. Biochemistry 47:5681-8. doi: 10.1021/bi8003044.

Corse E, Gottschalk RA, Krogsgaard M, Allison JP. 2010. Attenuated T cell responses to a high-potency ligand in vivo. PLOS Biol 8:e1000481. doi: 10.1371/journal.pbio.1000481.

Crocker JC, Grier DG. 1996. Methods of digital video microscopy for colloidal studies. J Colloid Interface Sci 179:298-310. doi: 10.1006/jcis.1996.0217.

Das J, Ho M, Zikherman J, Govern C, Yang M, Weiss A, et al. 2009. Digital signaling and hysteresis characterize ras activation in lymphoid cells. Cell 136:337-51. doi: 10.1016/j.cell.2008.11.051.

DeMond AL, Mossman KD, Starr T, Dustin ML, Groves JT. 2008. T cell receptor microcluster transport through molecular mazes reveals mechanism of translocation. Biophys J 94:3286-92. doi: 10.1529/biophysj.107.119099.

Domanski J, Stansfeld P, Sansom MP, Beckstein O. 2010. Lipidbook: a public repository for force-field parameters used in membrane simulations. J Membr Biol 236:255-8. doi: 10.1007/s00232-010-9296-8.

Dustin ML, Groves JT. 2012. Receptor signaling clusters in the immune synapse. Annu Rev Biophys 41:543-56. doi: 10.1146/annurev-biophys-042910-155238.

Ensign DL, Pande VS. 2009. Bayesian detection of intensity changes in single molecule and molecular dynamics trajectories. J Phys Chem B 114:280-92. doi: 10.1021/jp906786b.

Govern CC, Paczosa MK, Chakraborty AK, Huseby ES. 2010. Fast on-rates allow short dwell time ligands to activate T cells. Proc Natl Acad Sci USA 107:8724-9. doi: 10.1073/pnas.1000966107.

Grakoui A, Bromley SK, Sumen C, Davis MM, Shaw AS, Allen PM, et al. 1999. The immunological synapse: a molecular machine controlling T cell activation. Science 285:221-7. doi: 10.1126/science.285.5425.221.

Ha T, Ting AY, Liang J, Caldwell WB, Deniz AA, Chemla DS, et al. 1999. Single-molecule fluorescence spectroscopy of enzyme conformational dynamics and cleavage mechanism. Proc Natl Acad Sci USA 96:893-8. doi: 10.1073/ pnas.96.3.893.

Hawse WF, Champion MM, Joyce MV, Hellman LM, Hossain M, Ryan V, et al. 2012. Cutting edge: evidence for a dynamically driven T cell signaling mechanism. J Immunol 188:5819-23. doi: 10.4049/jimmunol.1200952.

Huang J, Zarnitsyna VI, Liu B, Edwards LJ, Jiang N, Evavold BD, et al. 2010. The kinetics of two-dimensional TCR and pMHC interactions determine T-cell responsiveness. Nature 464:932-6. doi: 10.1038/nature08944.

Huppa JB, Axmann M, Mortelmaier MA, Lillemeier BF, Newell EW, Brameshuber M, et al. 2010. TCR-peptide-MHC interactions in situ show accelerated kinetics and increased affinity. Nature 463:963-7. doi: 10.1038/nature08746.

Huse M, Klein LO, Girvin AT, Faraj JM, Li Q-J, Kuhns MS, et al. 2007. Spatial and temporal dynamics of T cell receptor signaling with a photoactivatable agonist. Immunity 27:76-88. doi: 10.1016/j.immuni.2007.05.017.

Irvine DJ, Purbhoo MA, Krogsgaard M, Davis MM. 2002. Direct observation of ligand recognition by T cells. Nature 419:845-9. doi: 10.1038/nature01076.

Iwashima M, Irving BA, van Oers NS, Chan AC, Weiss A. 1994. Sequential interactions of the TCR with two distinct cytoplasmic tyrosine kinases. Science 263:1136-9. doi: 10.1126/science.7509083.

Kaizuka Y, Douglass AD, Varma R, Dustin ML, Vale RD. 2007. Mechanisms for segregating T cell receptor and adhesion molecules during immunological synapse formation in Jurkat T cells. Proc Natl Acad Sci USA 104:20296-301. doi: 10.1073/pnas.0710258105.

Kaye J, Hsu ML, Sauron ME, Jameson SC, Gascoigne NR, Hedrick SM. 1989. Selective development of CD4+ T cells in transgenic mice expressing a class II MHC-restricted antigen receptor. Nature 341:746-9. doi: 10.1038/341746a0.

Krogsgaard M, Li QJ, Sumen C, Huppa JB, Huse M, Davis MM. 2005. Agonist/endogenous peptide-MHC heterodimers drive T cell activation and sensitivity. Nature 434:238-43. doi: 10.1038/nature03391.

Lin W-C, Yu C-H, Triffo S, Groves JT. 2010. Supported membrane formation, characterization, functionalization, and patterning for application in biological science and technology. In Current protocols in chemical biology 2. John Wiley \& Sons, Inc. pp. 235-69. doi: 10.1002/9780470559277.ch100131.

Majumdar DS, Smirnova I, Kasho V, Nir E, Kong X, Weiss S, et al. 2007. Single-molecule FRET reveals sugar-induced conformational dynamics in LacY. Proc Natl Acad Sci USA 104:12640-5. doi: 10.1073/pnas.0700969104.

Manz BN, Jackson BL, Petit RS, Dustin ML, Groves J. 2011. T-cell triggering thresholds are modulated by the number of antigen within individual T-cell receptor clusters. Proc Natl Acad Sci USA 108:9089-94. doi: 10.1073/ pnas.1018771108.

Matsui K, Boniface JJ, Steffner P, Reay PA, Davis MM. 1994. Kinetics of T-cell receptor binding to peptide/I-Ek complexes: correlation of the dissociation rate with T-cell responsiveness. Proc Natl Acad Sci USA 91:12862-6. doi: 10.1073/pnas.91.26.12862.

McKeithan TW. 1995. Kinetic proofreading in T-cell receptor signal transduction. Proc Natl Acad Sci USA 92:5042-6. doi: 10.1073/pnas.92.11.5042.

Newell EW, Ely LK, Kruse AC, Reay PA, Rodriguez SN, Lin AE, et al. 2011. Structural basis of specificity and cross-reactivity in T cell receptors specific for cytochrome c-I-E(k). J Immunol 186:5823-32. doi: 10.4049/jimmunol.1100197.

Nye JA, Groves JT. 2008. Kinetic control of Histidine-tagged protein surface density on supported lipid bilayers. Langmuir 24:4145-9. doi: 10.1021/la703788h.

Palmer E, Naeher D. 2009. Affinity threshold for thymic selection through a T-cell receptor-co-receptor zipper. Nat Rev Immunol 9:207-13. doi: 10.1038/nri2469.

Qi SY, Groves JT, Chakraborty AK. 2001. Synaptic pattern formation during cellular recognition. Proc Natl Acad Sci USA 98:6548-53. doi: 10.1073/pnas.111536798. 
Rabinowitz JD, Beeson C, Lyons DS, Davis MM, McConnell HM. 1996. Kinetic discrimination in T-cell activation. Proc Natl Acad Sci USA 93:1401-5. doi: 10.1073/pnas.93.4.1401.

Reboul CF, Meyer GR, Porebski BT, Borg NA, Buckle AM. 2012. Epitope flexibility and dynamic footprint revealed by molecular dynamics of a pMHC-TCR complex. PLOS Comput Biol 8:e1002404. doi: 10.1371/ journal.pcbi.1002404.

Schamel WW, Alarcon B. 2013. Organization of the resting TCR in nanoscale oligomers. Immunol Rev 251:13-20. doi: 10.1111/imr.12019.

Shaw AS, Dustin ML. 1997. Making the T cell receptor go the distance: a topological view of T cell activation. Immunity 6:361-9. doi: 10.1016/S1074-7613(00)80279-4.

Smith AW, Smoligovets AA, Groves JT. 2011. Patterned two-photon photoactivation illuminates spatial reorganization in live cells. J Phys Chem A 115:3867-75. doi: 10.1021/jp108295s.

Smoligovets AA, Smith AW, Wu H-J, Petit RS, Groves JT. 2012. Characterization of dynamic actin associations with T-cell receptor microclusters in primary T cells. J Cell Sci 125:735-42. doi: 10.1242/jcs.092825.

Stefanová I, Hemmer B, Vergelli M, Martin R, Biddison WE, Germain RN. 2003. TCR ligand discrimination is enforced by competing ERK positive and SHP-1 negative feedback pathways. Nat Immunol 4:248-54 doi: $10.1038 /$ ni895.

Tian S, Maile R, Collins EJ, Frelinger JA. 2007. CD8+ T cell activation is governed by TCR-Peptide/MHC affinity, not dissociation rate. J Immunol 179:2952-60.

Valitutti S, Muller S, Cella M, Padovan E, Lanzavecchia A. 1995. Serial triggering of many T-cell receptors by a few peptide-MHC complexes. Nature 375:148-51. doi: 10.1038/375148a0.

van der Merwe PA, Dushek O. 2011. Mechanisms for T cell receptor triggering. Nat Rev Immunol 11:47-55. doi: $10.1038 /$ nri2887.

Varma R, Campi G, Yokosuka T, Saito T, Dustin ML. 2006. T cell receptor-proximal signals are sustained in peripheral microclusters and terminated in the central supramolecular activation cluster. Immunity 25:117-27. doi: 10.1016/j.immuni.2006.04.010.

Weiss A, Littman DR. 1994. Signal transduction by lymphocyte antigen receptors. Cell 76:263-74. doi: 10.1016/0092-8674(94)90334-4.

Williams CB, Engle DL, Kersh GJ, Michael White J, Allen PM. 1999. A kinetic threshold between negative and positive selection based on the longevity of the T cell receptor-ligand complex. J Exp Med 189:1531-44. doi: 10.1084/jem.189.10.1531.

Xu Q, Lin WC, Petit RS, Groves JT. 2011. EphA2 receptor activation by monomeric Ephrin-A1 on supported membranes. Biophys J 101:2731-9. doi: 10.1016/j.bpj.2011.10.039.

Yin Y, Wang XX, Mariuzza RA. 2012. Crystal structure of a complete ternary complex of T-cell receptor, peptideMHC, and CD4. Proc Natl Acad Sci USA 109:5405-10. doi: 10.1073/pnas.1118801109.

Yokosuka T, Sakata-Sogawa K, Kobayashi W, Hiroshima M, Hashimoto-Tane A, Tokunaga M, et al. 2005. Newly generated T cell receptor microclusters initiate and sustain T cell activation by recruitment of Zap70 and SLP-76. Nat Immunol 6:1253-62. doi: 10.1038/ni1272.

Yu Y, Fay NC, Smoligovets AA, Wu HJ, Groves JT. 2012. Myosin IIA modulates T cell receptor transport and CasL phosphorylation during early immunological synapse formation. PLOS ONE 7:e30704. doi: 10.1371/ journal.pone.0030704.

Zhu C, Jiang N, Huang J, Zarnitsyna Vl, Evavold BD. 2013. Insights from in situ analysis of TCR-pMHC recognition: response of an interaction network. Immunol Rev 251:49-64. doi: 10.1111/imr.12016. 